\title{
The Application of A3 Aerial Photography in Island Surveying and Mapping
}

\author{
Ping WANG \\ South China Sea Institute of Planning and Environmental \\ Research, SOA \\ Guangzhou, China \\ 1903125@qq.com
}

\author{
Zheng WEI \\ South China Sea Institute of Planning and Environmental \\ Research, SOA \\ Guangzhou, China \\ weizheng0628@foxmail.com
}

\author{
Yu-chao SUN \\ South China Sea Institute of Planning and Environmental \\ Research, SOA \\ Guangzhou, China \\ 503598481@qq.com
}

\begin{abstract}
Based on the demand of island mapping, this paper analyzes the necessity of island mapping by aerial photogrammetry. Based on the key technical problems of island aerial photography, A3 aero-photography is used for island aerial photograph. This paper studies the route design, image processing and production process of the $A 3$ in the island surveying and mapping. The Dazhou island was taken as an example, the airline was designed to image, and the aerial imaging products were produced. This study can provide reference for the application of $\mathrm{A} 3$ aerial camera in island mapping.
\end{abstract}

Keywords-island aerial photography; A3 aerial camera; route design; aerial imaging products

\section{INTRODUCTION}

China's coastline is widely distributed and the number of islands is numerous. China's increasing emphasis on the protection and use of the island should be based on the timely investigation and assessment of the island's natural geographic information and development and utilization of the status. But most of the island area is small, simple structure, poor stability, the geographic information data and imagery acquisition is relatively difficult.

Current methods of island mapping include landing on-site monitoring, ship cruise monitoring, Statistical Survey, satellite remote sensing monitoring, aerial remote sensing monitoring, UAV aerial surveillance and other. Because of the fact that most of the islands are far from land, lacking ground control points, and unable to realize the on-site surveying work, the traditional methods of surveying are required to carry out artificial field measurement on the island. Therefore, the traditional method is difficult. Island measurement methods have been far from being able to meet the current needs of island surveys and surveillance.

Because of the spatial resolution, the acquisition of

\author{
Fan YANG ${ }^{*}$ \\ South China Sea Institute of Planning and Environmental \\ Research, SOA \\ Guangzhou, China \\ yangfan_whu@sina.cn \\ Ji-sheng ZENG \\ South China Sea Institute of Planning and Environmental \\ Research, SOA \\ Guangzhou, China \\ 969735@qq.com
}

image data, the revisiting period and the influence of weather, there are many limitations in the remote sensing image acquisition, so it is difficult to meet the needs of the island. Acquiring island image data by aerial photogrammetry had the advantages such as real-time image transmission, high-risk area detection, low cost, high resolution, flexible and so on. Airborne receivers can acquire multi-temporal island images in real-time using a variety of sensors such as high-resolution CCD cameras, laser radar and infrared detectors.

Therefore, this paper is based on the requirement of island mapping and the application of A3 aerial photography in island mapping, focusing on A3 aerial photography's measurement mode, and to solve the A3 aerial photography in the tilt measurement mode data processing and marine mapping applications. In this paper, taking DaZhou Island as an example, the A3 aerial photography is applied to the island mapping, and the island aerial imaging, data processing and the DTM, DEM, DOM, DLG and other geographic information products are completed. In this paper, the application of the A3 surveying and mapping of A3 aerial camera is realized, and the technical methods and operation flow of the A3 surveying and mapping are summarized.

\section{ANALYSIS ON THE SURVEYING AND MAPPING REQUIREMENTS OF ISLANDS}

\section{A. The Needs of Island Mapping}

In order to grasp the natural reefs of the geographical information and development and utilization of the status quo to achieve the island dynamic monitoring, island ecological protection and island development and utilization, etc. The needs of island mapping include:

(1) Island basic geographic information data. The basic geographic information of island mainly includes island DEM, DTM, DSM, DOM and DLG. 
(2) Land use / cover change. The island development and utilization survey shall acquire the status quo of the use of buildings, roads, vegetation and public facilities on the island, and analyze the current situation of island land use according to the time series remote sensing images at certain intervals.

\section{B. The Necessity of Aerial Photogrammetry}

The islands have the characteristics of small target, fast distribution and fast change, resulting in the following problems in the process of obtaining the basic geographic information and development and utilization status in island mapping:

(1) Landed island mapping not only the existence of heavy workload, high cost and low efficiency, and difficult to obtain the island's panoramic image data.

(2) The spatial resolution of high-resolution remote sensing satellites at home and abroad is mostly $0.5 \mathrm{~m}$ or more, while the area of many micro-islands is less than $500 \mathrm{~m} 2$, which is limited by island area and remote sensing image space when using remote sensing satellite to acquire island image data Resolution, it is difficult to interpret island information by using island image data obtained from high spatial resolution remote sensing image.

(3) The high resolution remote sensing satellite is difficult to obtain under different tide conditions of the island image, and the data acquisition is vulnerable to cloudy and rainy weather conditions.

Therefore, the use of aerial photogrammetry technology for island mapping can acquire the high resolution image data of the whole island and acquire the island image of arbitrary tide in real time.

\section{Key Technical Issues}

Taking into account the needs of island mapping, the application of aerial photogrammetry technology, the following key technical issues to be considered:

(1) The area of island in the South China Sea is relatively small, and the image resolution of the low spatial resolution is difficult to meet the requirements of the island topography. Therefore, the image data in the island mapping needs a higher space resolution.

(2) Some islands in China have ownership disputes, and some islands in the East China Sea and the South China Sea are not clearly defined or occupied by other countries. Due to their rights, land surveying and orthorectic imaging over the island airspace cannot be carried out, therefore, island mapping needs to use sideways model of aerial remote sensing.

(3) Because there is no control point on the island, in order to achieve a higher accuracy in the orthorectification of the image, the DGPS (Differential Global Positioning System) and PPP (Precise Point Positioning, Precise Point Positioning) were used to perform aerial triangulation calculations without control points to ensure the accuracy of aerial photogrammetry products.

Aiming at the key problems to be solved in the above-mentioned island surveying and mapping, the A3 aerial photogrammetry is used in the paper to conduct island aerial photogrammetry and produce island geographic information products.

\section{APPLICATION OF A3 IN ISLAND SURVEYING AND MAPPING}

Considering the particularity and key technical problems of island mapping, A3 aerial photogrammetry has high spatial resolution and can achieve centimeter resolution at $5 \mathrm{~km}$ altitude, and can acquire high precision and high spatial resolution remote sensing image of island area efficiently. A3 Aerial photogrammetry has three imaging mode. A3 image data processing software package LightSpeed can be in the absence of ground control points under the conditions of the data for automated processing and product geographic information products.

Therefore, in order to solve the key technical problems in island mapping, the A3 aerial photogrammetry is used for the island aerial photogrammetry in view of the specific needs and special circumstances of island mapping. A3 Aerial Data Processing System is a fully automated terminal aerial surveying system that automates data processing in the absence of ground control points. After the flight of A3 aerial system, you can also get a variety of data products: DOM, DEM, DSM and tilt mapping products.

\section{A. Technical Parameters and Operating Principles}

The physical map of A3 aerial camera was shown in Figure 1.

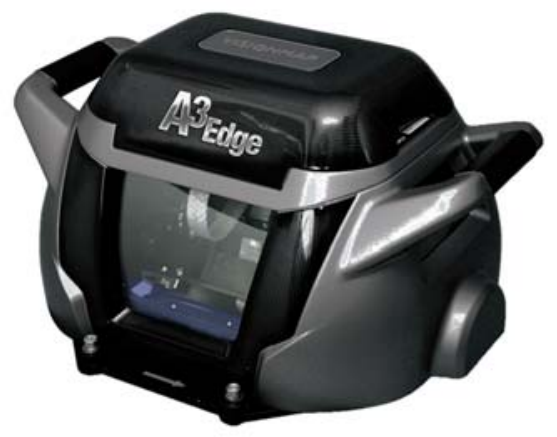

Figure 1. A3 aerial camera physical map

The technical parameters of A3 aerial camera was shown in Table 1.

TABLE 1. THE TECHNICAL PARAMETERS OF A3

\begin{tabular}{|l|l|}
\hline Parameter & Value \\
\hline Focal length & $300 \mathrm{~mm}$ \\
\hline Pixel size & $7.4 \mu \mathrm{m}$ \\
\hline Imaging frequency & 8 images per second \\
\hline Number of pixels & 16 million per image \\
\hline The maximum swing angle & $109^{\circ}$ \\
\hline SLF size & $73000 * 9600$ \\
\hline Weight & $42 \mathrm{~kg}$ \\
\hline
\end{tabular}

A3 aircraft carrying dual-frequency GPS, it can record the location information of each sub-image in real-time, and obtain precise position accuracy of $2 \sim 20 \mathrm{~cm}$ by sub-base station or post-precision single point positioning technology.

In the imaging of A3 camera the forward and side overlap should be more than $60 \%$. So, the same feature can appear on a large number of images. By using a large number of overlapped sub images, multi-visual and 
multi-baseline matching techniques can be used to obtain a large number of matching data, and a dense triangulation can be constructed. At the same time a large number of redundant results were obtained, the point accuracy is close to the actual value in a higher precision than other aerial digital cameras.

\section{B. Operating Mode and Applications}

A3 aircraft operating modes include: orthophoto metric mode, oblique three-dimensional measurement mode and unilateral side-scan measurement mode, as shown in Figure 2.

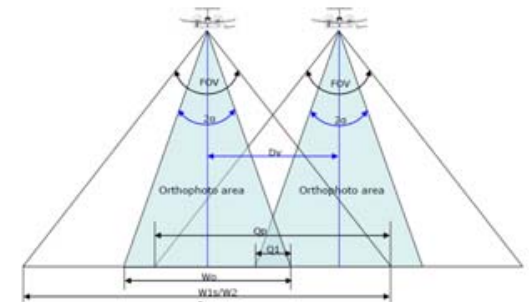

(A) orthophoto metric mode

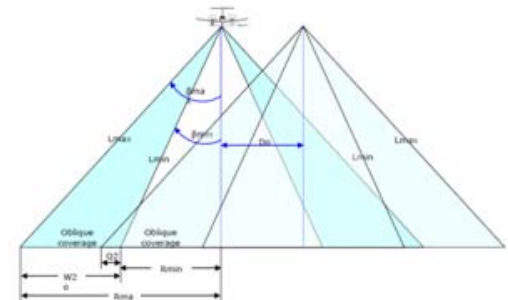

(C) Unilateral side-scan measurement mode

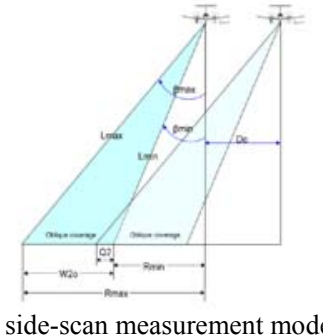

Figure 2. A3 aircraft operating mode
In the island mapping, the orthophoto metric measurement mode (a) can be applied to the island orthophoto production, thematic map production. The oblique three-dimensional measurement mode (b) can be applied to island DSM, DTM and DEM products. Side scan measurement mode (c) is often used in the case of the island cannot be normal flight, for example, when in aerial imaging of the islands with unclear ownership, the airplane cannot image directly above the island, using the unilateral side-scan model of A3 aerial camera can get the images in the distance of $10 \mathrm{~km}$ away from the islands. It greatly improved the efficiency in the acquisition of aerial image.

\section{A3 AERIAL IMAGING AND DATA PROCESSING}

\section{A. Airline Design and Image Acquisition}

A3 aerial camera to obtain high-quality aerial remote sensing image is based on route design and flight control. The route is developed for the flight quality elements and their sub-elements in the aerial photography quality model, including the ground sampling interval, aerial scale, flight height, aerial photography division of the division, route design, photography time and the flight parameters. The design of the above parameters plays a decisive role in the imaging of A3 aerial photography.

1) Flight height and speed

In the case where the ground resolution is fixed, the altitude $H$ is determined by the ratio of the focal length $f$ of the aerial camera to the size of the single pixel $a$.

$$
H=f \times G S D / a
$$

Where GSD is the ground sampling distance.

The speed GS at the time of imaging of A3 is determined by the GSD and the swept speed of the linear array, which can be expressed as

$$
G S=G S D / C T
$$

Where, $C T$ is the camera linear array recording interval.

The maximum and minimum speeds in flight are allowed to vary within a certain range of the optimum flight speed. If the actual flight speed exceeds the maximum speed, the cell will be stretched and the image resolution will be reduced. If the speed is lower than the minimum speed, the image will be repeated sampling, increasing the amount of flight data.

2) The image overlaps

In A3 aerial photogrammetry, the forward overlap is generally controlled between $60 \%$ and $65 \%$, the side overlap is generally controlled between $30 \%$ and $35 \%$. The image overlap is used to ensure the stereo observation, image matching and model connection. The forward overlap determines the base height ratio at imaging. The side overlap determines the distance between adjacent routes.

3) Airline laying

In addition to satisfying aerial photography related technical requirements and above-mentioned route design parameters, the route layout of island reef aerial imaging also needs to consider the shape characteristics, coastline and spatial distribution of coastal zone and island reef. Therefore, the island reef aerial photography, the route should be considered the layout of the coastline shape characteristics, the direction of flight should be consistent with the distribution of the island, using parallel to the coastline of the fixed course.

\section{B. Image Processing and Producing}

A3 aerial camera aerial triangulation solution processing methods include DGPS mode, PPP mode, DGPS and GCPs mode, PPP and GCPs mode. As the control points cannot be laid on island, the solution of A3 aerial images is mainly used DGPS or PPP mode.

1) Image quality inspection and pretreatment

FlightViewer software was used to check the original images, and the courses with large sail angle were selected, and the airline for later data processing was selected. And the GrafNav software was used to process the original GPS data.

2) Air triangulation

The division of air three encrypted partition is according to the airline. The flight speed software was used to calculate airborne triangulation encryption.

Select the solution for the latter part of the navigation 
zone, tasks and sweep range, you can set up the software through the air Triangulation LightSpeed task. Free image adjustment of the images involved in the free network adjustment to restore the relative position between the photos.

After the completion of the free network adjustment, the quality of aerial triangulation is checked, mainly including the connecting line and corresponding points.

3) The results produced

DSM / DTM are generated according to the aerial triangulation, and the DEM are produced based on the DSM result using TerraSolid software to eliminate non-ground point cloud.

Using the edited DEM and aerial triangulation results, the original image is orthorectified. The model orthorectimages of the whole survey area are seamlessly stitched by automatically generated mosaic lines, then the DOM are generated, the splicing lines, color, uniform light of DOM are checked.

\section{APPLICATION OF A3 IN ISLAND MAPPING}

The paper introduces the application process of the image acquisition, data processing, DOM and DTM images of the A3 using the oblique three-dimensional measurement mode on the Dazhou island.

Airline Design

The imaging airline design parameters of Dazhou Island are shown in the table 2, the airline layout is shown in figure 3.
TABLE. 2 VALUE OF AIRLINE PARAMETERS

\begin{tabular}{|l|l|}
\hline Airline parameters & Value \\
\hline Resolution & $8.5 \mathrm{~cm}$ \\
\hline Flight height & $3000 \mathrm{~m}$ \\
\hline Flight speed & $130 \mathrm{knot}$ \\
\hline Airline spacing & $1100 \mathrm{~m}$ \\
\hline Flying area & $13 \mathrm{~km}^{2}$ \\
\hline Number of routes & $10 / 9$ \\
\hline Length of routes & $10.5 \mathrm{~km} / 11.5 \mathrm{~km}$ \\
\hline
\end{tabular}

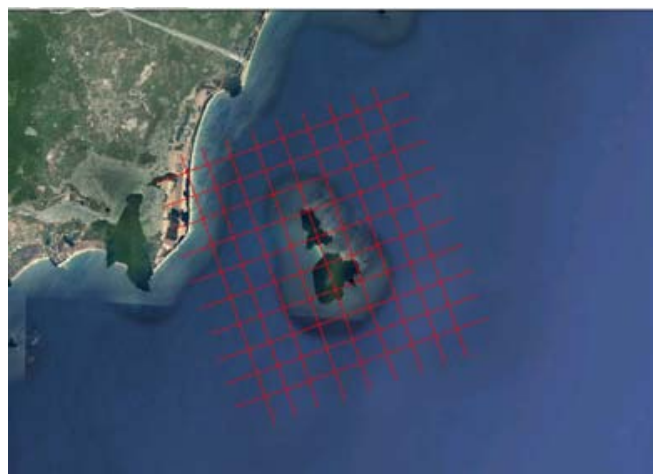

Figure 3. Figure of airline layout

\section{A. A3 Aircraft Surveying and Mapping Results}

Through the above route design and image acquisition, the DTM and DOM of Dazhou Island were produced according to the A3 image processing flow.

The DTM, DOM, DEM are shown in figure 4. The three-dimensional point cloud model is shown in figure 5 .

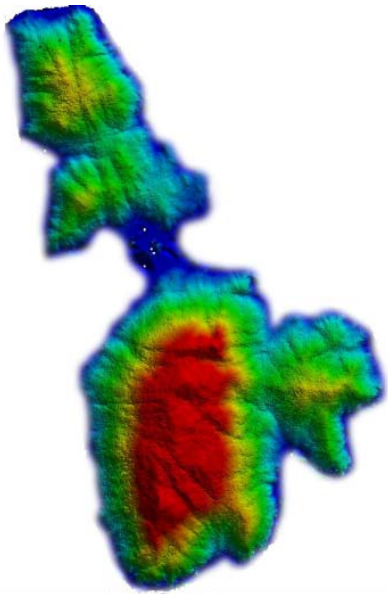

(a) DTM

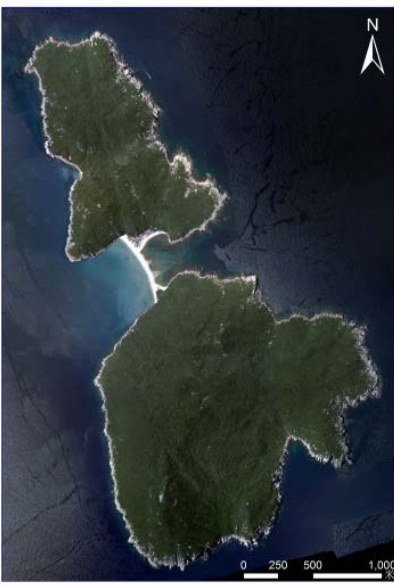

(b) DOM

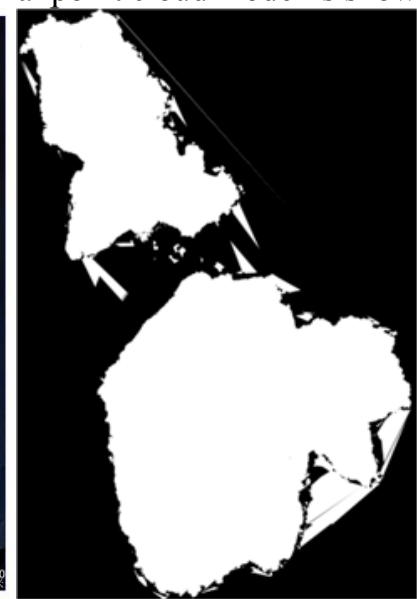

(c) DEM

Figure 4. The image productions of Dazhou Island

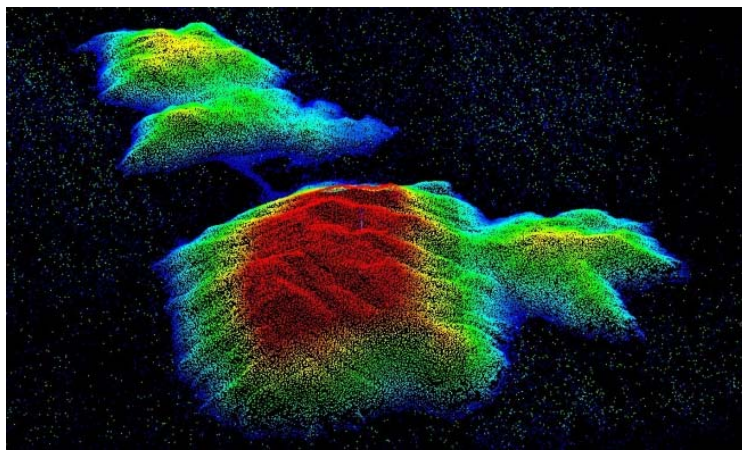

Figure 5. The 3-D point cloud mode 


\section{CONCLUSIONS AND SUGGESTIONS}

According to A3 data acquisition and processing in the process of island imaging, the high resolution aerial images of islands can be acquired, and the products such as DTM, DOM and DEM can be obtained. The efficiency of image acquisition and data processing are high, and product quality is high, can well meet the needs of the island mapping.

The following questions should be paid attention in the $\mathrm{A} 3$ aerial mapping applications in islands:

(1) Using A3 to aerial photography in the coastal zone of the island, using the CORS station as the base station, the uncontrolled aerial triangulation adjustment and the DOM accuracy can meet the requirement of aerodynamics of 1:2000 scale aerial photography.

(2) According to the geographical distribution of the island and the characteristics of the status quo, the number of control points should be appropriately increased. As the island image contains large area of water that will affect the image matching accuracy. To ensure the accuracy of post-air triangulation, you can increase the control point in the island to improve the accuracy of results and improve work efficiency.

(3) This paper can provide a comprehensive and reliable solution for the application of A3 aerial photography in the island area.

\section{REFERENCES}

[1] HUANG Yao, ZHANG Caixia, XI Ge, et al. "An Application Study of A3 Camera in Island and Coastal Zone Survey". HYDROGRAPHIC SURVEYING AND CHARTING, vol 36, Sep.2016, pp.79-82, DOI: 10.3969/j.issn.1671-3044.2016.05.02 0 .

[2] Zhang Xinxin, Wang Dong, $\mathrm{Hu} \mathrm{Ke}$, et al. "Stereo Mapping Based on A3 SLF Image". Urban Geotechnical Investigation \& Surveying, vol 5, Oct.2015, pp.83-85.

[3] Zhou Shuwang. "Study on the Principle and Method of Obtai ning High Precision Results Without Control Points". Science and Technology Innovation Herald, vol 24, 2014, pp.24-27, DOI:10.16660/j .cnki .1674-098x.2014.24.002.

[4] JIANG Chunhua. "Experimental Research on Plan of Setting out Control Point for A3 Digital Camera in the Condition of Complex Topography". Bulletin of Surveying and Mapping, vol 5, 2015, pp.84-86, DOI: 10.13474 /j.cnki.11-2246.2015.01 52.

[5] Li Haolin, Li Chong, Huang Ruijin, et al. "An Overlap Chec k Method for A3 Digital Aerial Camera". Remote Sensing In formation, vol 30, Dec.2015, pp.58-62, DOI:10.3969/j.issn.100 $0-3177.2015 .06 .011$

[6] WANG Xin, JIANG Ting, ZHANG Baoyin, et al. "Optimizati on Strategy for Digital Aerial Photography Plan of Coastal Z one and Island". Journal of Geomatics Science an d Technolo gy, vol 28, Mar.2011, pp190-193, DOI: 10 .3969/ j.issn.1673$6338.2011 .03 \quad 009$

[7] Wang Haiyun, Yu Rusong, Miao Shiwei, et al. "The Utilizati on of Photogrammetry Technology in Island Topographic Surv ey". HYDROGRAPHIC SURVEYING AND CHARTING, vol 31, Mar.2011, pp.45-48.

[8] Su Yongqi. "Study on the Orthogonal Projection Production P rocess Based on A3 System". Science and Technology Innova tion Herald, vol 20, 2015, pp.36, DOI:10.16660/j.cnki.1674-09 8x.2015.20.011.

[9] Teng Huizhong, Shen Jiashuang, Liu Min, et al. "Views on Construction of Aerophotogrammetric System for Hydrographi c Surveying and Charting". HYDROGRAPHIC SURVEYING AND CHARTING, vol 35, Jul.2015, pp.11-15. DOI: 10.3969 j. issn.1671-3044.2015. 04.003.
[10] Zhang Liang, Teng Huizhong, Ouyang Yongzhong. "Operation Instructions for Island Aerial Photography in the South Chin a Sea". HYDROGRAPHIC SURVEYING AND CHARTING, vol 34, Nov. 2014, pp.63-66. DOI: 10.3969 /j. issn.1671-3044. 2014.06.017. 